\title{
Electrooptical modulation in multiple quantum well hetero nipi waveguides
}

\author{
Thirstrup, C.; Robson, P. N.; Li Kam Wa, P.; Pate, M. A.; Button, C. C.; Roberts, J. S.
}

Published in:

Journal of Lightwave Technology

Link to article, DOI:

$10.1109 / 50.285324$

Publication date:

1994

Document Version

Publisher's PDF, also known as Version of record

Link back to DTU Orbit

Citation (APA):

Thirstrup, C., Robson, P. N., Li Kam Wa, P., Pate, M. A., Button, C. C., \& Roberts, J. S. (1994). Electrooptical modulation in multiple quantum well hetero nipi waveguides. Journal of Lightwave Technology, 12(3), 425-429. https://doi.org/10.1109/50.285324

\section{General rights}

Copyright and moral rights for the publications made accessible in the public portal are retained by the authors and/or other copyright owners and it is a condition of accessing publications that users recognise and abide by the legal requirements associated with these rights.

- Users may download and print one copy of any publication from the public portal for the purpose of private study or research.

- You may not further distribute the material or use it for any profit-making activity or commercial gain

- You may freely distribute the URL identifying the publication in the public portal 


\title{
Electrooptical Modulation in Multiple Quantum Well Hetero nipi Waveguides
}

\author{
C. Thirstrup, P. N. Robson, P. Li Kam Wa, M. A. Pate, C. C. Button, and J. S. Roberts
}

\begin{abstract}
An optical intensity modulator based on multiple quantum well hetero (MQW-H) nipi waveguides is reported. In the low optical power regime $\left(\sim 10^{-5} \mathrm{~W}\right)$, the modulator exhibits an extinction ratio in excess of 100:1 at low drive voltage $(4 \mathrm{~V})$ and 5-B attenuation. Modelling and experimental results of the time response of the waveguide modulator are presented, and it is shown that the response is limited by the $R C$ time constant of the drive circuit and the photocurrent charging up a dielectric capacitance. The modelling shows that with a loss penalty of $\sim 1.5$ $\mathrm{dB}$, the speed of the present waveguide modulator is limited solely by the $R C$ time constant that would allow modulation speeds of $\sim 100 \mathrm{ps}$.
\end{abstract}

\section{INTRODUCTION}

$\mathbf{M}$ ULTIPLE quantum well hetero (MQW-H) nipi structures have been shown to exhibit large optical nonlinearities [1]-[5]. The structures consist of alternating MQW pin and nip diodes and the physical mechanism is a selfphoto-induced modulation of built-in electric fields arising from space charge induced separation of photoexcited carriers and a long electron-hole recombination time. Most work until now on nipi structures have focussed on optical based modulators, although a few publications have treated electrooptical properties of nipi photodetectors and nipi electrooptical modulators [6]-[8]. A promising application of these devices is a hybrid configuration involving a detector combined with an electrooptical modulator for all-optical switching [7].

This paper focusses on an electrooptical modulator based on the quantum confined Stark effect [9] in GaAs/GaAlAs MQW-H nipi waveguides. The intrinsic regions in each MQW pin and nip diode of the modulator are narrow $(\sim 0.14 \mu \mathrm{m}$ wide) and high electric fields can be obtained across the quantum wells at low applied voltages. In order to obtain a similar confinement of the guided light in a MQW pin diode waveguide modulator, the intrinsic region must be 5-7 times wider and larger voltages are required for similar electric fields in the quantum wells. The MQW-H nipi waveguide modulator reported here therefore exhibits a larger electro-absorption response than a waveguide modulator based on a MQW pin diode structure. In the low optical power regime $\left(\sim 10^{-5} \mathrm{~W}\right)$, extinction ratios in excess of 100:1 have been demonstrated

Manuscript received April 22, 1993; revised October 14, 1993.

P. N. Robson, M. A. Pate, C. C. Burton, and J. S. Roberts are with the Department of Electronic and Electrical Engineering, University of Sheffield, Sheffield SI 3JD, England.

C. Thirstrup is with the Mikroelektronik Centret, Technical University of Denmark, 2800 Lyngby, Denmark.

P. L. K. Wa is with the Center for Research in Electro-Optics and Lasers, University of Central Florida, Orlando, FL 32826

IEEE Log Number 9216340. with 4-V drive voltage and attenuation of $5 \mathrm{~dB}$. In Section II, the device operation and the modulation depths obtained in the MQW-H nipi waveguide modulator are reported. A model is presented in Section III which describes the time response of the modulator, and finally in Section IV, experimental results of the time response are presented and compared with the model.

\section{Device Operation AND Modulation DePTH}

\section{A. Electronic Band Structure of A Biased MQW-H nipi}

Due to the large optical nonlinearities in MQW-H nipi structures the absorption is highly sensitive to changes in incident optical power [1]-[5]. This behavior also affects the electrooptical properties of the structures. At increasing optical powers, the photoexcited carriers cause a screening in the electric field across the quantum wells and reduce the quantum confined Stark effect. This is illustrated in Fig. 1 where the conduction band energy $E_{\mathrm{c}}$ (upper solid curves), valence band energy $E_{v}$ (lower solid curves), the electron quasi-Fermi energy $E_{F C}$ (dashed curves) and the hole quasiFermi energy $E_{F V}$ (dotted curves) are shown as function of distance $z$ perpendicular to the MQW-H nipi structure in the case of (a) zero bias voltage and zero optical power; (b) nonzero bias voltage $V_{b}$ and zero optical power; and (c) nonzero bias voltage $V_{b}$ and nonzero optical power $P_{i n}$. The calculations were obtained from a model reported in [10].

Fig. 1. shows two periods of a MQW-H nipi structure comprising six $100 \AA$ GaAs quantum wells $/ 100 \AA$ GaAlAs $\mathrm{Ga}_{0.7} \mathrm{~A} 1_{0.3}$. As barriers in each intrinsic region of the pins and nips. The quasi-Fermi energies of the electrons and the holes are assumed to coincide at the contacts of the structure (not shown in Fig. 1). The voltage drop along the $z$-direction is equal to the change in the electron (or hole) quasi-Fermi energy multiplied by the (negative) electron charge $-q$. In Fig. 1(b), (c), the total voltage drop is $-q \Delta E_{\mathrm{FC}}=-q \Delta E_{\mathrm{FV}} \simeq-3.3 V$. The optical power in Fig. 1(c) has been set equal to $P_{\text {in }}=100 \mu \mathrm{W}$.

In thermal equilibrium (see Fig. I(a)), the quasi-Fermi energies for the electrons and the holes coincide throughout the structure. When the voltage is applied (see Fig. 1(b)), the quasi-Fermi energies are separated and drop over the reverse biased pins and only change slightly over the forward biased nips. The fact that most of the voltage applied to the MQW$\mathrm{H}$ nipi structure is dropped over the reverse biased pins is an advantage in terms of optical modulation: only reverse biased diodes contribute to the desired quantum confined 


\section{E}
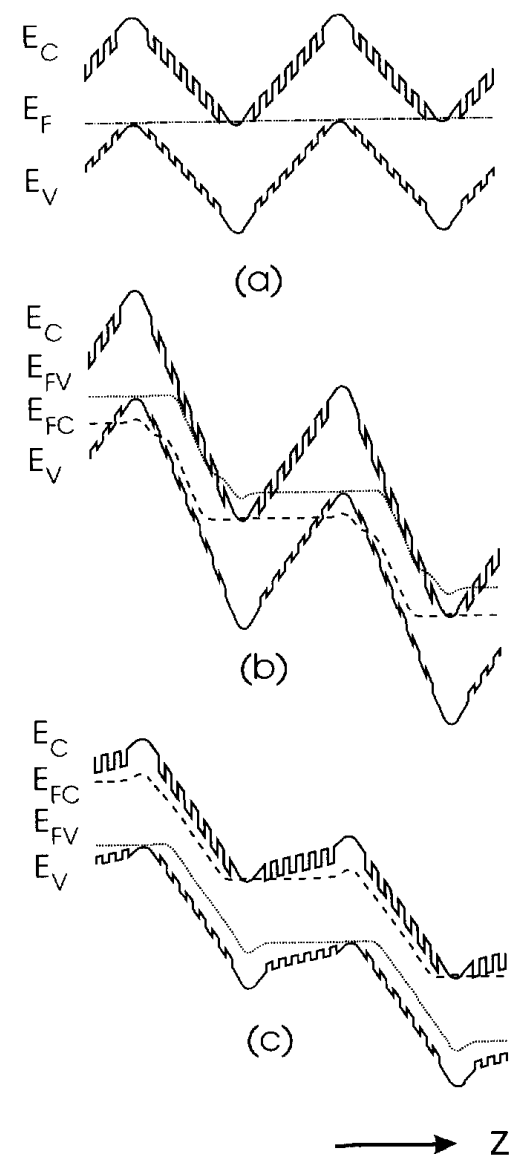

Fig. 1. Conduction band energy $E_{r}$ ( (upper solid curves), valence band energy $E_{V}$ (lower solid curves), electron quasi-Fermi energy $E_{\mathrm{F}}$ : (dashed curves) and hole quasi-Fermi energy $E_{\mathrm{FV}}$ (dotted curves) as function of distance $z$ perpendicular to the MQW-H nipi structure; (a) zero bias voltage and zero optical power; (b) nonzero bias voltage and zero optical power; and (c) nonzero bias voltage and nonzero optical power.

Stark shift of the absorption edge toward a longer wavelength [9]. Forward biased nips cause an undesirable shift of the absorption edge in the opposite direction.

At higher optical powers (tens of microwatts), the selfphoto-induced modulation becomes important. The large number of photoexcited carriers created in the intrinsic MQWregions of the pins and the nips cause a screening of the electric field across the quantum wells as shown in Fig. I(c). At higher optical powers, it is therefore necessary to apply larger voltages in order to achieve the same modulation of the absorption coefficient.

\section{B. Experimental Results}

The structure used for electro-optical modulation was grown by atmospheric pressure MOVPE and is identical to that reported in [5]. The MQW-H nipi region consists of alternating a $200 \AA$ wide $\mathrm{Ga}_{0.7} \mathrm{Al}_{0.3}$ As $p^{+}$-region $\left(p \approx 2 \times 10^{18} \mathrm{~cm}^{-3}\right)$, an intrinsic MQW-region containing 6 periods of $100 \AA \mathrm{GaAs}$ quantum wells and $100 \AA \mathrm{Ga}_{0.7} \mathrm{Al}_{0.3}$ As barriers and a $200-\AA$ wide $\mathrm{Ga}_{0.7} \mathrm{Al}_{0.3}$ As $n^{+}$-region $\left(n \approx 2 \times 10^{18} \mathrm{~cm}^{-3}\right.$ ). Additionally, $50-\AA$ undoped $\mathrm{Ga}_{0.7} \mathrm{Al}_{0.3} \mathrm{As}$ buffer layers were grown on

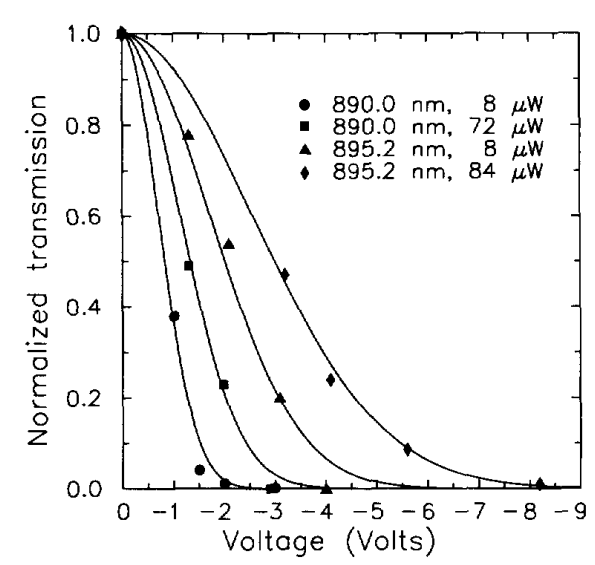

Fig. 2. Normalized transmission through the waveguide modulators as function of applied voltage, (a) wavelength $\lambda=890.0 \mathrm{~nm}$ and input optical power $P_{\mathrm{in} 1}=8 \mu \mathrm{W}$ (circle symbols); (b) $\lambda=890.0 \mathrm{~nm}$ and $P_{\mathrm{in}}=72 \mu \mathrm{W}$ (square symbols); (c) $\lambda=895.2 \mathrm{~nm}$ and $P_{\mathrm{in}}=8 \mu \mathrm{W}$ (triangular symbols); and (d) $\lambda=895.2 \mathrm{~nm}$ and $P_{\text {in }}=84 \mu \mathrm{W}$ (diamond symbols). The solid curves are best fits obtained using (1) in the text.

each side of the MQW-regions. The MQW-H nipi structure is repeated $N=7$ times giving a total thickness of $1.14 \mu \mathrm{m}$, and it is sandwiched between a $1 \mu \mathrm{m} \mathrm{Ga}{ }_{0.8} \mathrm{Al}_{0.2} \mathrm{As} p^{+}$-region and a $1 \mu \mathrm{m} \mathrm{Ga} \mathrm{ma}_{0.8} \mathrm{Al}_{0.2} \mathrm{As} n^{+}$-region on the top of an $n^{+}$-type GaAs substrate. This waveguide configuration provides single mode guiding for wavelengths larger than $\sim 850 \mathrm{~nm}$.

$\mathrm{A} \mathrm{Au} / \mathrm{AuZn}$ alloy was evaporated on the top of the structure to provide an ohmic metal mask for the waveguides and $\mathrm{AuGe}$ was evaporated on the back. Ridge waveguides with a width of $W_{\mathrm{WG}}=4 \mu \mathrm{m}$ were fabricated using reactive ion etching to remove the top $p^{+}$-region outside the waveguide metal mask. In order to reduce the total capacitance of the devices, $W=$ $100-\mu \mathrm{m}$-wide mesa's surrounding the waveguides were made using a standard wet-etch. Gold wires were bonded to the waveguides to provide electrical contacts. For the experiments reported here, $L=1.8$-mm-long waveguides were used.

The measurements of the optical modulation were performed by coupling light from a styryl 9 dye laser into and out of the waveguides using microscope objectives. Silicon photodetectors were used to monitor the input and output optical powers. Fig. 2 shows the normalized transmission through the waveguide modulators as function of voltage (negative in relation to the top $p^{+}$-region) applied for different wavelengths $\lambda$ and input optical powers $P_{\text {in }}$; (a) $\lambda=890.0 \mathrm{~nm}$, $P_{\text {in }}=8 \mu \mathrm{W}$ (circle symbols); (b) $\lambda=890.0 \mathrm{~nm}, P_{\text {in }}=72 \mu \mathrm{W}$ (square symbols); (c) $\lambda=895.2 \mathrm{~nm}, P_{\text {in }}=8 \mu \mathrm{W}$ (triangular symbols); and (d) $\lambda=895.2 \mathrm{~nm}, P_{\text {in }}=84 \mu \mathrm{W}$ (diamond symbols). The solid lines are best fits to the experimental data assuming the following dependence between the normalized transmission $T$ and the applied voltage $V_{b}$;

$$
\begin{aligned}
T & =\exp \left[-\Delta \alpha\left(V_{b}\right) L\right] \\
\Delta \alpha\left(V_{b}\right) & =a V_{b}^{2}
\end{aligned}
$$

where $\Delta \alpha\left(V_{b}\right)$ is the change in the absorption coefficient and $a$ is a fitting parameter and where it is assumed that the modulation is based on the quantum confined Stark effect which is quadratic in voltage [9]. The fitting parameters a 
for the solid curves in Fig. 2 are (a) 1.0, (b) 0.39, (c) 0.17 , and (d) $0.077 \mathrm{~cm}^{-1} V^{-2}$, respectively. The fitting curves are observed to follow the normalized transmission; however, at voltages corresponding to transmission less than $\sim 10 \%$, the measured data lie somewhat below the fitting curves.

Room temperature photoluminescense (PL) measurements performed on the MQW-H nipi structures showed a maximum PL-intensity of $\sim 864 \mathrm{~nm}$ and the two operating wavelengths 890.0 and $895.2 \mathrm{~nm}$ reported in Fig. 2 are sufficiently below the absorption edge to yield low optical losses. This observation was confirmed by measuring the absorption and scattering losses in the waveguides using sequential cleaving. The loss measurements were performed at low input optical powers $(\sim$ $\left.10^{-5} \mathrm{~W}\right)$. The results are $12 \mathrm{~cm}^{-1}$ at $890.0 \mathrm{~nm}$ and $6 \mathrm{~cm}^{-1}$ at $895.2 \mathrm{~nm}$. The corresponding total losses in the waveguide modulator samples are $\alpha_{0} L=9$ and $5 \mathrm{~dB}$, respectively. At increasing optical powers, the photoexcited carriers tend to screen the electric fields across the quantum wells and this causes a slight reduction in the absorption losses (see [5]).

It is observed in Fig. 2 that very large modulation depths can be achieved when a few voltages are applied to the waveguides. For instance, at a wavelength of $890.0 \mathrm{~nm}$ and an optical power of $8 \mu \mathrm{W}$, a voltage of $-3 \mathrm{~V}$ is sufficient to modulate the output optical power below the resolution in Fig. 2. With the sensitivity and noise level of the photodetectors used in the experiment, it is found that the extinction ratio is in excess of 100:1. Similarly, at the longer wavelength $895.2 \mathrm{~nm}$ and at an optical power of $8 \mu \mathrm{W}$, a voltage of $-4 \mathrm{~V}$ is sufficient to yield an extinction ratio in excess of 100:1. For similar attenuation and drive voltage, these modulation depths are better than those achieved in GaAs/GaAlAs MQW pin diode waveguides [11] and comparable to the best reported results in waveguide modulators based on $\mathrm{GaAs} / \mathrm{GaAlAs}$ superlattices [12] and single quantum well graded index separate confined heterostructures [13]. However, at higher optical powers the $\mathrm{MQW}-\mathrm{H}$ nipi waveguide modulator has a poorer performance with respect to drive voltage. When the input optical power is increased to $84 \mu \mathrm{W}$ at $895.2 \mathrm{~nm}$, the drive voltage for $100: 1$ extinction ratio is increased to $>8 \mathrm{~V}$. As described in Section II, this is due to the photoexcited carriers that screen the electric fields. When the optical power approached $1 \mathrm{~mW}$, the drive voltages for 100:1 extinction ratio were observed to approach or exceed the break down voltage of the current MQW-H nipi waveguide samples $(\sim 10 \mathrm{~V})$.

\section{TIME RESPONSE OF THE MODULATOR}

\section{A. A Model}

In order to model the time response of the MQW-H nipi waveguide modulator, the equivalent circuit diagram in Fig. 3 of one period of a MQW-H nipi structure can be used. The diagram illustrates a nip with a diode $D_{1}$, a photocurrent density generator $J_{L 1}$ and a capacitance $C_{1}$. The nip is connected in series to a pin with the similar components $D_{2}, J_{L 2}$ and $C_{2}$ and to a bias circuit with resistance $R$ and voltage $V_{b}$.

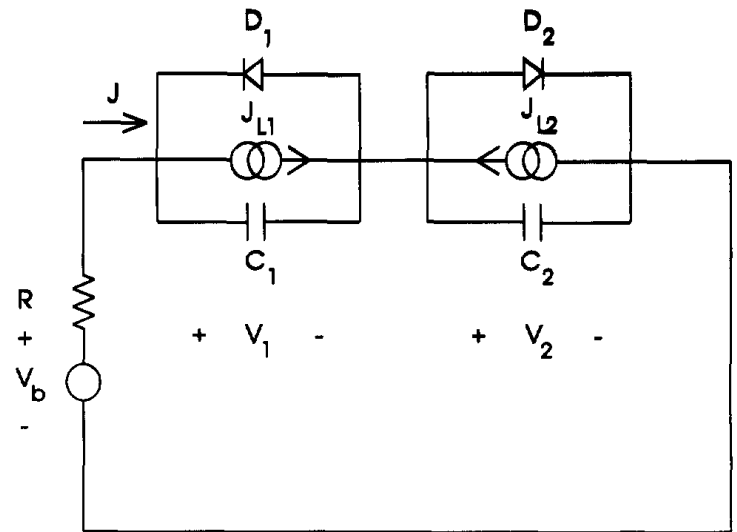

Fig. 3. Equivalent circuit diagram of a MQW-H nipi waveguide connected to a resistive bias circuit. The different components are described in the text.

Applying Kirchoff's laws to the diagram in Fig. 3 yields the following two coupled differential equations:

$$
\begin{aligned}
& C_{1} \frac{d V_{1}}{d t}-J_{s}\left(e^{-q V_{1} / \mathrm{mkT}}-1\right) A+ \frac{n_{1} V_{1}+n_{2} V_{2}}{R} \\
&=\frac{1}{R} V_{b}(t)-J_{L 1} A \quad \text { (2a) } \\
& C_{2} \frac{d V_{2}}{d t}+J_{s}\left(e^{q V_{2} / \mathrm{mkT}}-1\right) A+\frac{n_{1} V_{1}+n_{2} V_{2}}{R} \\
&=\frac{1}{R} V_{b}(t)+J_{L 2} A \quad \text { (2b) }
\end{aligned}
$$

where $V_{1}$ and $V_{2}$ are the voltages across the nip and the pin diode, respectively, $m$ is the diode ideality factor which for recombination dominated current is equal to $2, J_{s}$ is the dark current density, $A$ is the area of the diode, and $n_{1}$ and $n_{2}$ are the number of nip and pin diodes, respectively. To zero order in absorption, the photocurrent densities $J_{L 1}$ and $J_{L 2}$ can be written:

$$
J_{L 1}=J_{L 2}=J_{L}=\frac{q \eta}{\hbar \omega} P_{\text {in }}\left\{1-e^{-\alpha_{0} L}\right.
$$

where $\eta$ is the internal quantum efficiency, $h \omega$ is the photon energy, $P_{\text {in }}$ is the input optical power, $L$ is the length of the waveguide and $\alpha_{0}$ is the unexcited absorption coefficient.

Assuming that the $n_{1}$ reverse biased pins solely contribute to the voltage induced absorption change (see Section II.A), the output optical power can be expressed:

$$
\begin{aligned}
P_{\text {out }} & =P_{\text {in }} e^{-\left[\alpha_{0}+\Delta \alpha\left(n_{2} V_{2}\right)\right] L} \\
\Delta \alpha\left(n_{2} V_{2}\right) & =a\left(n_{2} V_{2}\right)^{2}
\end{aligned}
$$

where $a$ is an empirical parameter defined in (1), and $n_{2} V_{2} \cong$ $V_{b}$ in steady state where the total current density $J$ is negligible.

The two coupled differential equations are readily solved using a fourth order Runge-Kutta method. In an optical modulation experiment, $V_{b}(t)$ is typically a step function and in this case the initial conditions $V_{1}(t=0+)$ and $V_{2}(t=0+)$ are determined solving (2) for $V_{b}(t=0-)$ and setting $d / d t=0$.

\section{B. Experimental Results}

In order to test the time response of the MQW-H nipi waveguide modulators, a voltage pulse generator with square 


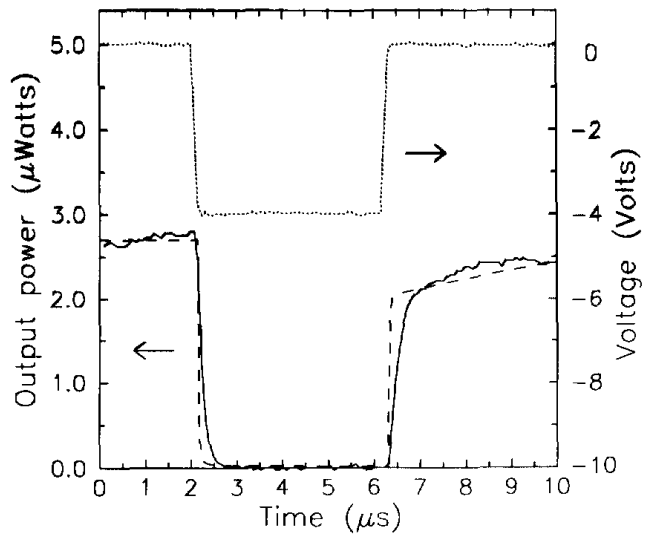

Fig. 4. Time response of a MQW-H nipi waveguide modulator at $\lambda=895.2$ $\mathrm{nm}$ and with input optical power $P_{\mathrm{in}}=8 \mu \mathrm{W}$. The bias voltage $V_{b}=-4.0$ $\mathrm{V}$ (dotted curve) is applied to the waveguide at $t=2.2 \mu \mathrm{s}$ and returns to zero at $t=6.3 \mu \mathrm{s}$. The solid curve is measured output optical power $P_{\text {out }}$ and the corresponding dashed curve is obtained from the model as described in the text.

output pulses and a $R=1 \mathrm{k} \Omega$ load resistor were connected to the waveguides. The polarity of the pulses was negative in relation to the top $p^{+}$-region implying that 4 pins were negatively biased and 3 nips were positively biased.

Fig. 4 shows the time response of the waveguide modulator at $\lambda=895.2 \mathrm{~nm}$ where the bias voltage $V_{b}=-4.0 \mathrm{~V}$ (dotted curve) is applied to the waveguide at $t=2.2 \mu \mathrm{s}$ and returns to zero at $t=6.3 \mu \mathrm{s}$. The fall time and rise time of the voltage pulses are $0.2 \mu \mathrm{s}$. The input optical power is $P_{\mathrm{in}}=8$ $\mu \mathrm{W}$ corresponding to the case with triangular symbols in Fig. 2. The solid curve in Fig. 4 is the measured output optical power $P_{\text {out }}$ and the dashed curve is the corresponding result obtained from the model described in the previous section using the following parameters: $P_{\text {in }}=8 \mu \mathrm{W}, V_{b}=-4.0$ $\mathrm{V}$ (step function), $n_{1}=3, n_{2}=4, \alpha_{0}=6 \mathrm{~cm}^{-1}$ (see section II.B), $\eta=1, \hbar \omega=1.39 \mathrm{eV}$ (for $\lambda=895.2 \mathrm{~nm}$ ), $L=1.8 \mathrm{~mm}, m=2, J_{s}=1.71 \times 10^{-5} \mathrm{~A} / \mathrm{cm}^{2}$ (see [5]) and $A=\mathrm{WL}=1.8 \times 10^{-3} \mathrm{~cm}^{2}$. A fitting parameter of $a=0.39 \mathrm{~cm}^{-1} \mathrm{~V}^{-2}$ was used in the model to get best fit to the experimental result. This value is larger than $a=0.17$ $\mathrm{cm}^{-1} \mathrm{~V}^{-2}$, which is used to fit the triangular symbols in Fig. 2 , but underestimates the modulation depths for transmission less than $10 \%$ (see Section II.B). For the capacitances $C_{1}$ and $C_{2}$, the following expression is used:

$$
C_{1,2}=C_{D}+C_{S}\left(V_{1,2}\right)
$$

where $C_{D}$ is the dielectric capacitance that has been determined to be $7.5 \times 10^{-8} \mathrm{~F} / \mathrm{cm}^{2}$ [5] and $C_{S}$ is the (forward voltage) space charge capacitance. For the sake of simplicity, it is assumed that $C_{S}=0$ for reverse bias, and $C_{S}=10 \times 10^{-8}$ $\mathrm{F} / \mathrm{cm}^{2}$ for forward bias which is a typical value for weakly forward-biased diodes [14].

It is observed from the experimental result (the solid curve in Fig. 4) and predicted by the model (the dashed curve in Fig. 4) that the rise time consists of an initial "fast" response that is followed by a slower response towards steady state. Approximate analytical expressions for the rise times and the fall times can be obtained from the model. When the voltage is applied, $P_{\text {out }}$ drops exponentially towards zero with a $1 / \mathrm{e}$ time constant approximately given by;

$$
\tau_{f} \cong 2 R \frac{C_{1} C_{2}}{n_{1} C_{2}+n_{2} C_{1}} A .
$$

This formula is easily derived by considering the equivalent circuit in Fig. 3 and replacing the diodes $D_{1}$ and $D_{2}$ by resistors $R_{1}$ and $R_{2}$. In this case, the coupled differential equations (2) can be solved analytically and by assuming $R_{1}, R_{2} \gg R,(5)$ is obtained.

Using $C_{1}=17.5 \times 10^{-8} \mathrm{~F} / \mathrm{cm}^{2}$ and $C_{2}=7.5 \times 10^{-8} \mathrm{~F} / \mathrm{cm}^{2}$ and the above mentioned parameters yields, $\tau_{f}=5 \times 10^{-8}$ s. The model predicts a faster fall time than observed in Fig. 4 due to a nonideal step-voltage applied to the waveguides and parasitic capacitances in the bias circuit. The voltage is ramped in $0.20 \mu \mathrm{s}$, which is similar to a measured 1/e fall time of the output optical power of $0.15 \pm 0.04 \mu \mathrm{s}$.

When the voltage returns to zero at $t=6.3 \mu \mathrm{s}$, two different time constants are observed. Initially, the output optical power returns to $\sim 70 \%$ of the steady state level, which is determined approximately by the ratio of the capacitances $n_{2} C_{1} /\left(n_{1} C_{2}+n_{2} C_{1}\right)$. The time constant is

$$
\tau_{r 1} \cong \tau_{f}
$$

where $\tau_{f}$ is given by (5) implying that, $\tau_{r 1}=5 \times 10^{-8}$ s. For the same reason as above, the experimental value is larger. Due to the space charge capacitance, the forwardbiased diodes have larger capacitance than the reverse-biased diodes: $C_{1}>C_{2}$. Assuming that, $C_{1}=C_{2}$, the output optical power returns to $57 \%$ instead of $70 \%$ of the steady state level. However, normally $C_{1}$ is appreciably larger than $C_{2}$ and the results are closer to $70 \%$ than $57 \%$.

From $\sim 70 \%$ to $100 \%$ of the steady state level, the output optical power is observed to decay on a much slower time scale (see Fig. 4), which is determined by the photocurrent charging up the dielectric capacitor $C_{D}$. The $10 \%-90 \%$ rise time of the residual output optical power can be approximated by the following expression derived in [5]:

$$
\tau_{r 2}=\frac{C_{D} A}{J_{L}} \frac{\operatorname{mkT}}{q} \log _{e}\left[\frac{\left(J_{L} / J_{S}\right)^{9 / 10}-1}{\left(J_{L} / J_{S}\right)^{1 / 10}-1}\right] .
$$

Using the parameters from above yields, $\tau_{r 2}=5 \times 10^{-6} \mathrm{~s}$, which is comparable to the experimental result (see Fig. 4).

Experimentally, the "slow" time constant $\tau_{r 2}$ has been observed to decrease approximately inverse proportional to the input optical power $P_{\text {in }}$ [5]. This also agrees with (7) where the logarithmic term varies slowly with $J_{L}$ and since $J_{L} \propto P_{\text {in }}$, the time constant $\tau_{r 2} \propto 1 / P_{\text {in }}$. With the time resolution in the current experiment, it was not possible to see any change in the "fast" part of the time response $\tau_{f}$ and $\tau_{r 1}$ with increasing optical power. From the model, no change or only a small change is expected since the only excitation dependent parameters for $\tau_{f}$ and $\tau_{r 1}$ are the space charge capacitances (see (5)) and they only have a modest variation with excitation [14]. A comparison between the expressions (5)-(7) of the time constants and the corresponding figures derived from the numerical model described in Section III. A 
shows that for input optical powers between $10^{-6}$ and $10^{-4}$ $\mathrm{W}$, the deviations are within $50 \%$ for $\tau_{f}, \tau_{r 1}$ and $\tau_{r 2}$. The expressions (5)-(7) can therefore be used to give a rough estimate of the magnitude of the time constants.

It has been observed by the authors that by applying $1 \mathrm{MHz}$ square pulses with a duty cycle of $50 \%$ to the waveguide modulators, the output optical power is able to respond with an unchanged low level, but with the high level reduced to $\sim 70 \%$ of the steady state value which corresponds to the point in Fig. 4, where the initial "fast" response governed by $\tau_{r 1}$ is replaced by the "slow" response governed by $\tau_{r 2}$. Thus, with $30 \%$ or $1.5-\mathrm{dB}$ loss penalty it was possible to achieve optical modulation with a time response limited by the $R C$ time constants $\tau_{f}$ and $\tau_{r 1}$. An upper bound on this loss penalty is $43 \%$ or $2.4 \mathrm{~dB}$ corresponding to the case where, $C_{1}=C_{2}$.

In the present device configuration, it should be possible to achieve considerable improvement in the time response. Equations (5) and (6) show that $\tau_{f}$ and $\tau_{r 1}$ are determined by the drive load resistance $R$ and the area of the waveguide $A$. If the width of the MQW-H nipi region is reduced from $W=100 \mu \mathrm{m}$ to the width of the waveguide $W_{\mathrm{WG}}=4 \mu \mathrm{m}$ and if the load resistance $R$ is reduced from $1 k \Omega$ to $50 \Omega$, a reduction in $\tau_{f}$ and $\tau_{r 1}$ of $\frac{4}{100} \times \frac{50}{1000}=0.002$ is obtained. The corresponding time constant is, $0.002 \times 5 \times 10^{-8} \mathrm{~s}=100$ ps. The residual time response $\tau_{r 2}$ (see (7)) is only affected by the reduction in the area of the waveguide that yields, $\frac{4}{100} \times 5 \times 10^{-6} \mathrm{~s}=200 \mathrm{~ns}$. This estimate shows that if $\mathrm{GHz}$ modulation is desirable, it must be based exclusively on the time constants $\tau_{f}$ and $\tau_{r 1}$. For the case shown in Fig. 4 , this implies additional losses of $\sim 1.5 \mathrm{~dB}$.

\section{CONCLUSION}

An electrooptical intensity modulator based on MQW-H nipi structures has been analyzed. The modulator is competitive with other electrooptical modulators for optical powers in the microwatt regime. At optical powers of about $10^{-5} \mathrm{~W}$, extinction ratios in excess of $100: 1$ with $4-\mathrm{V}$ drive voltage and attenuation of $5 \mathrm{~dB}$ have been demonstrated. At higher optical powers, larger voltages are needed to produce the same extinction ratio and when the optical powers approach $1 \mathrm{~mW}$, the drive voltage performance of the MQW-H nipi modulator becomes worse than modulators based on superlattices or quantum wells. The time response consists of two time constants: one is determined by the $R C$ time constant of the drive circuit, which for the present device configuration could be reduced to $\sim 100 \mathrm{ps}$, and one is determined by the photocurrent charging up a dielectric capacitor and limited to the nanosecond or microsecond regime. With a loss penalty of approximately $1.5 \mathrm{~dB}$, modelling and experimental results show that the operation of the modulator can be based exclusively on the fast part of the time response.

\section{REFERENCES}

[1] A. Kost, E. Garmire, A. Danner and P. D. Dapkus, "Large optical nonlinearities in a GaAs/AIGaAs hetero nipi structure," Appl. Phys. Lett., vol. 54, pp. 637-639, 1988.

[2] A. Kost, "Performance characteristics of hetero n-i-p-i dtructures," Optical and Quantum Electronics, vol. 22, pp. S187-S200, 1990.

[3] H. Ando, H. Iwamura, H. Oohashi and H. Kanbe, "Nonlinear absorption in nipi-MQW structure," Proc. 35th Conf. Japan Soc. Appl. Phys., p. 966, 1988.

[4] H. Ando, H. Iwamura, H. Oohashi and H. Kanbe, "Nonlinear absorption in nipi-MQW structure," IEEE J. Quantum Electron., vol. QE-25, pp. 2135-2141, 1989.

[5] C. Thirstrup, P. N. Robson, P. Li Kam Wa, M. A. Pate, C. C. Button and J. S. Roberts, "Optical nonlinearities in GaAs/GaAlAs multiple quantum well hetero-nipi waveguides," IEEE J. Quantum Electron, vol. QE-28, pp. 864-874, 1992.

[6] P. Kiesel, P. Riel, N. Linder, J. N. Miller and G. H. Döhler, "Linear photoresponse, optical switching and optical bistability in reverse biased nipi doping superlattices," Superlattices and Microstructures, vol. 6, pp. $363-368,1989$.

[7] G. H. Döhler, P. Kiesel, Meißmeier, G. Pototzky, P. Riel, J. Fouquet, J. N. Miller, G. Trott and J. Williamson, "Responsivity, detectivity and high-frequency performance of reverse biased photoconductive $n-i-p-i$ detector", Quantum Well and Superlattice Physics II, Eds. F. Capasso, G. H. Döhler, J. N. Schulman, Newport Beach, USA, SPIE, vol. 943, pp. $129-135,1988$.

[8] X. Wu, K. H. Gulden, M. Thomas, J. S. Smith, J. R. Whinnery, S. Malzer, P. Kiesel, M. Kneissl and G. H. Döhler, "Hetero- nipi band filling modulator with laterally interdigital contacts made by shadow mask molecular beam epitaxy regrowth," Appl. Phys. Lett., vol, 62, pp. 152-153, 1993.

[9] D. A. B. Miller, D. S. Chemla, T. C. Damen, A. C. Gossard, W. Wiegmann, T. H. Wood and C. A. Burrus, "Electric field dependence of optical absorption near the band gab of quantum well structures," Phys. Rev. B, vol. 32, pp. 1043-1060, 1985.

[10] C. Thirstrup, "Novel electro-optical phase modulator based on GaInAs/InP modulation-doped quantum-well structures," Appl. Phys. Lett., vol. 61, pp. 2641-2643, 1992.

[11] T.H. Wood, "Multiple quantum well (MQW) waveguide modulators," J. Lightwave Technol., vol. 6, pp. 743-757, 1988.

[12] A. L. Moretti, D. J. Vezzetti, F. A. Chambers, K. A. Stair and G. P. Devane, "GaAs/Al $\mathrm{Al}_{x} \mathrm{Ga}_{1}$ As superlattice waveguide absorption modulators with very low drive voltage," IEEE Photon. Technol. Lett., vol. 4, pp. 576-579, 1992

[13] D. Moss, D. Landheer, A. Delage, F. Chatenoud and M. Dion, "Laser compatible waveguide electroabsorption modulator with high contrast and low operating voltage in GaAs/AlGaAs," IEEE Photon. Technol. Lett., vol. 3, pp. 645-647, 1991.

[14] J. J. Liou, F. A. Lindholm and J. S. Park, "Forward-voltage capacitance and thickness of $\mathrm{p}-\mathrm{n}$ junction space-charge regions," IEEE Trans. Electron Devices, vol. ED-34, pp. 1571-1579, 1987.

C. Thirstrup, photograph and biography not available at the time of publication.

P. N. Robson, photograph and biography not available at the time of publication.

P. Li Kam Wa, photograph and biography not available at the time of publication.

M. A. Pate, photograph and biography not available at the time of publication.

C. C. Button, photograph and biography not available at the time of publication.

J. S. Roberts, photograph and biography not available at the time of publication. 